\title{
Situaciones meteorológicas con impacto en las operaciones en el aeropuerto de Madrid-Barajas Adolfo Suárez
}

\author{
Darío Cano Espadas ${ }^{1}$ (dcanoe@aemet.es) \\ Alejandro Méndez Frades² (amendezf@aemet.es) \\ ${ }^{1}$ AEMET / Delegación Territorial en Madrid \\ ${ }^{2}$ AEMET / Dirección de Planificación, Estrategia y Desarrollo Comercial
}

\section{RESUMEN}

Se describen situaciones meteorológicas que causan impacto en las operaciones del aeropuerto de Madrid-Barajas Adolfo Suárez.

PALABRAS CLAVE: impactos; meteorología aeronáutica; Madrid-Barajas Adolfo Suárez; brisas de montaña; dipolo orográfico; cizalladura; nieblas; operaciones aeroportuarias; tormentas.

\section{INTRODUCCIÓN}

Cuando se habla de «situaciones meteorológicas de impacto» inconscientemente se nos vienen a la cabeza grandes catástrofes como por ejemplo, inundaciones, vendavales, nevadas, olas de calor o de frío, etc. Sin embargo, lo que la sociedad entiende como «situación de buen tiempo», en ciertas actividades socioeconómicas, puede tener una incidencia nada desdeñable. Pongamos por caso el ámbito de la aviación: las aeronaves tan solo pueden operar por debajo de una determinada temperatura, están expuestas a recurrentes cambios de configuración durante los días de verano o incluso su volumen de ocupación puede verse mermado por las restricciones debidas al ruido u otro tipo de contaminantes químicos, muy común en los días invernales apacibles gobernados por un anticiclón.

En numerosas ocasiones, el mayor o menor impacto vendrá determinado por la hora de ocurrencia, la persistencia y el tráfico en el aeropuerto. De esta forma, un cambio de configuración o la aparición/disipación de nieblas tendrán una mayor incidencia en la hora de mayor afluencia, a diferencia de otras con menor actividad.

\section{EL AEROPUERTO DE MADRID-BARAJAS ADOLFO SUÁREZ: ENTORNO MESOESCALAR}

El aeropuerto de Madrid-Barajas Adolfo Suárez (en adelante, LEMD) se halla situado en la cuenca alta del Tajo, paralelo al río Jarama y muy cercano a la confluencia con el río Henares. Su altitud es de unos 600 metros sobre el nivel del mar, localizándose en el fondo de los valles que forman la sierra de Guadarrama en el sistema Central y las sierras de Albarracín en el sistema Ibérico. Esta configuración orográfica favorece el establecimiento de un eficaz sistema de brisas de montaña y una frecuente incidencia por las nieblas de río. Además de la instrumentación reglamentaria con la que cuenta LEMD, el entorno mesobeta está equipado 
con numerosas estaciones meteorológicas automáticas, un radar, una estación de radiosondeos y un perfilador de viento y temperatura (figura 1).

LEMD posee cuatro pistas, paralelas dos a dos. Dos pistas orientadas en la dirección N-S (18/36 L, 18/36 R) y las otras dos orientadas en la dirección NE-SW (14/32 L, 14/32 R). Como los dos pares de pistas son contiguos, las cabeceras de entrada o salida solo pueden ser cuatro: las dos 18 y las dos 32. Así, habrá dos configuraciones posibles: la configuración norte, en la que los aviones llegan por las cabeceras 32 y salen por las cabeceras 18 y la configuración sur, en la que las salidas se efectúan por las cabeceras 32 y las llegadas por las 18 (figura 2).

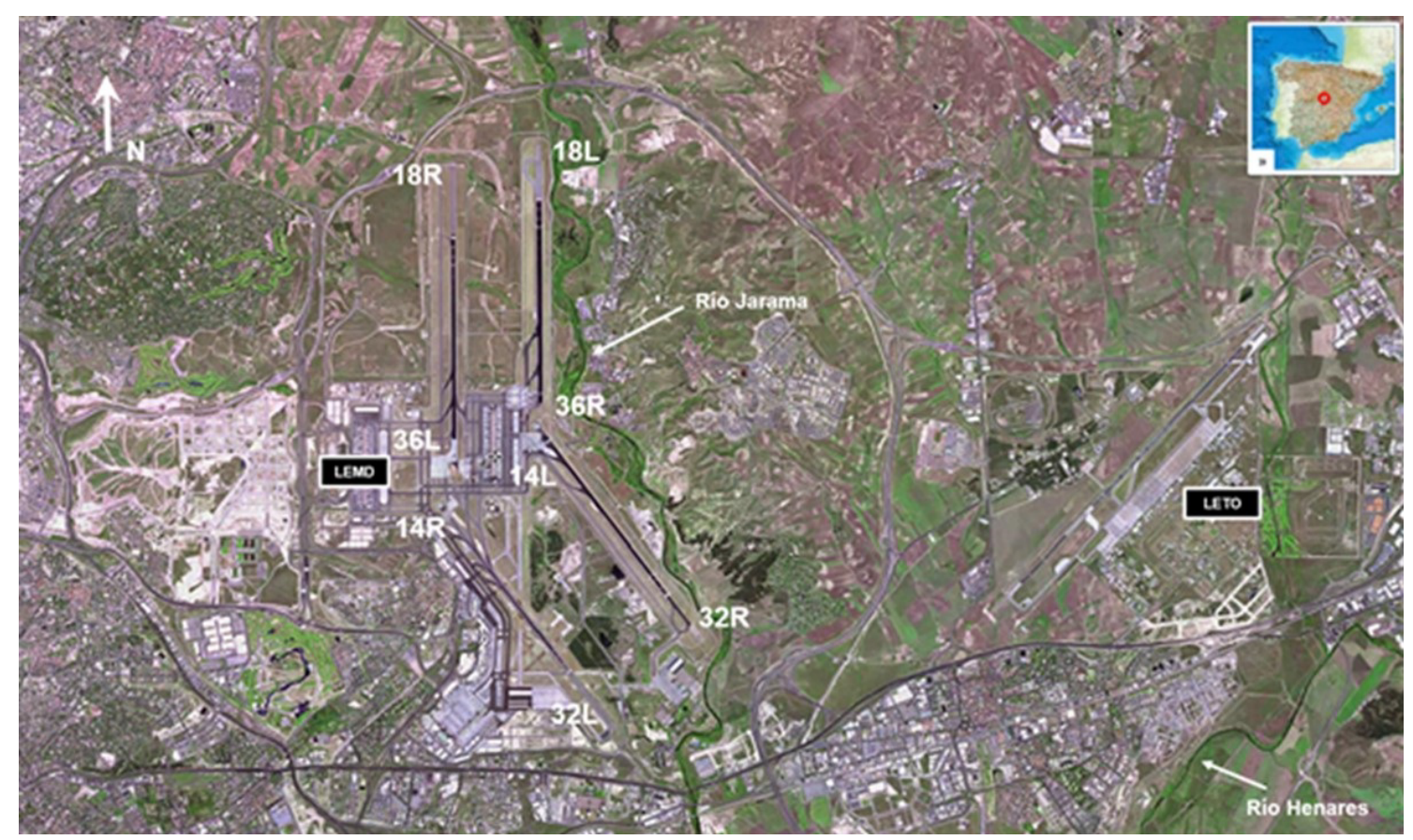

Figura 1. Mapa de LEMD donde se indican las pistas y el nombre de las cabeceras. Véase la cercanía a la confluencia de los ríos Jarama y Henares y a la base aérea de Torrejón de Ardoz (LETO). Fuente: Iberpix.

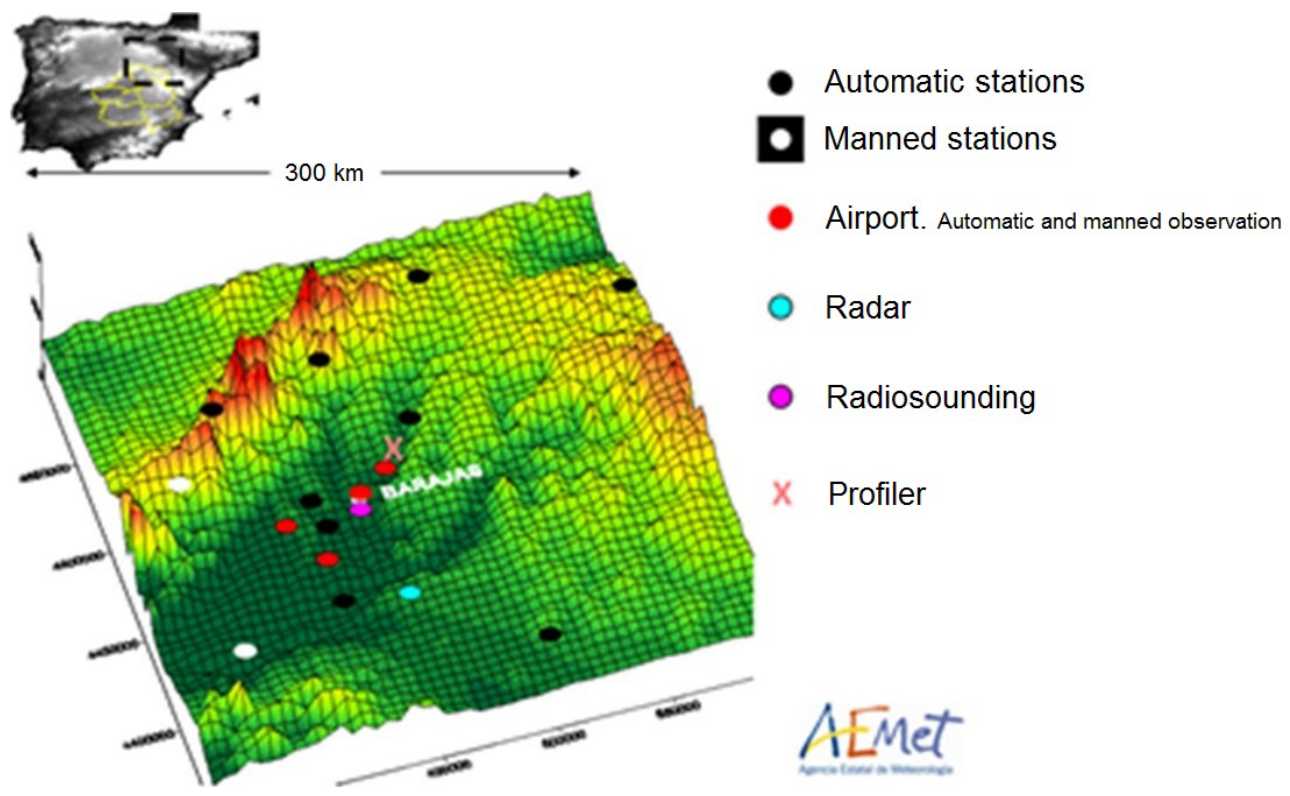

Figura 2. Entorno mesobeta de LEMD y localización de las principales fuentes de datos meteorológicos. Fuente: AEMET. 


\section{CAMBIOS DE CONFIGURACIÓN Y BRISAS DE MONTAÑA}

El cambio de configuración de las pistas supone un gran impacto en las operaciones, sobre todo cuando tienen lugar en una fase del día en la que hay un gran volumen de tráfico. Así, un cambio de configuración en estas condiciones hace que todas las aeronaves que están a la espera de tomar tierra sean reconfiguradas a fin de tomar posiciones adecuadas para llevar a cabo su operación con la mayor seguridad y eficiencia posible. Este hecho es de especial impacto en las grandes aeronaves de procedencia transatlántica que llegan con las tripulaciones cansadas y los depósitos de combustible casi vacíos.

La configuración preferente de LEMD es la norte que solo se cambia si se producen o se esperan vientos medios o rachas con componente en cola superiores a $10 \mathrm{kt}$.

Hay que tener en cuenta que un cambio a configuración sur reduce la capacidad de asumir tráfico ya que provoca una mayor contaminación acústica. Esto se debe a la cercanía de lugares residenciales y a que el aeropuerto está sujeto a una regulación sobre ruidos.

Por otra parte, las brisas de montaña constituyen un fenómeno habitual en LEMD (CANO, PALACIO, TÉLLEZ BEATRIz et al., 2001). En condiciones de estabilidad, cielos despejados y cuando las condiciones sinópticas de viento no son relevantes, se produce un drenaje catabático de componente N-NE por la noche, desde las zonas altas al fondo de los valles y un viento anabático, de componente S-SW durante el día, desde los valles hacia las zonas altas.

Si bien los catabáticos no tienen especial incidencia en la operatividad debido a su baja intensidad (no supera los $5 \mathrm{kt}$ ), ocurre lo contrario con los anabáticos ya que, durante los meses de mayor insolación (mayo a octubre), pueden superar los $10 \mathrm{kt}$, pudiendo incluso alcanzar los $15 \mathrm{kt}$. Por este motivo, LEMD se ve obligado a operar en configuración sur durante las horas centrales del día.

En la figura 3 se muestra el modelo conceptual de su formación y evolución. En efecto, como consecuencia del ciclo diurno de la insolación, en las primeras horas de la mañana la cara sur del sistema Central está inicialmente iluminada. Posteriormente, pasa a estarlo las del sistema Ibérico (serranía de Cuenca). Este hecho es el causante de la intensificación y cambio de dirección (de S a SW) de los anabáticos.

El comportamiento descrito anteriormente hace que, durante las horas de máxima intensidad (en torno a las 12 UTC) las pistas 18/36 tengan más viento de componente en cola que las 14/32. En estas últimas, el viento tiene más componente transversal.

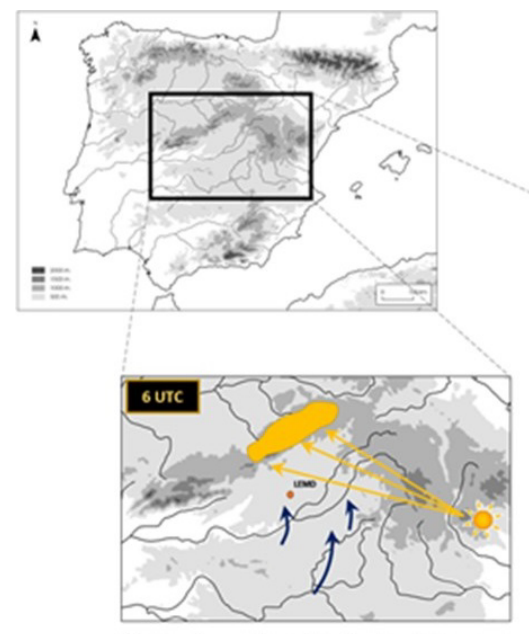

En las primeras horas del dia, el sol sale por el E, calentando las laderas de la Sierra de Guadarrama (amarillo), induciéndose de forma espontánea un viento mesoescalar anabático. del S (azul).

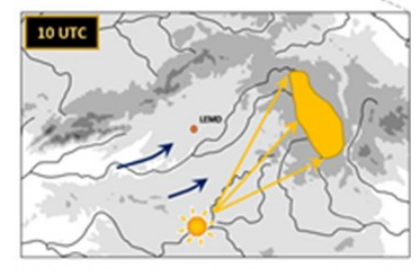

Hacia las horas centrales, el sol avanza progresivamente hacia el $\mathrm{S}$, calentando las laderas de la serrania de Cuenca (amarillo). induciendo un giro del viento anabático a W- SW (azul).
Figura 3.

Modelo conceptual de la formación de vientos anabáticos durante los meses de mayor insolación en LEMD.

La parte más problemática es la predicción del role del viento de S a SW a efectos de establecer la configuración más adecuada. Fuente: elaboración propia. 
Tan importante es predecir el inicio del viento anabático superior a $10 \mathrm{kt}$ como su final, es decir, la ventana temporal en la que LEMD se encontrará en condiciones de viento de configuración sur. En este sentido, el TAF realizado para LEMD debe hacer alusión a este fenómeno. El TREND de viento es más difícil de precisar pero, al menos, debería ser coherente con el mensaje TAF. Ahora bien, dado que el pronóstico de aeródromo TAF y TREND va dirigido al dato del METAR que, en el caso de LEMD, toma el dato de viento de la cabecera 32L, puede ocurrir que otras cabeceras superen el umbral de los $10 \mathrm{kt}$ de cola sin que tenga que ser incluida en el METAR (figura 4a).

Como se aprecia en la figura 4, el final del episodio (viento del S superior a $10 \mathrm{kt}$ de cola) es mucho más incierto que en su aparición. Por este motivo la permanencia de un pronóstico de configuración sur, aun cuando no se está observando, suele ser más habitual.

a)

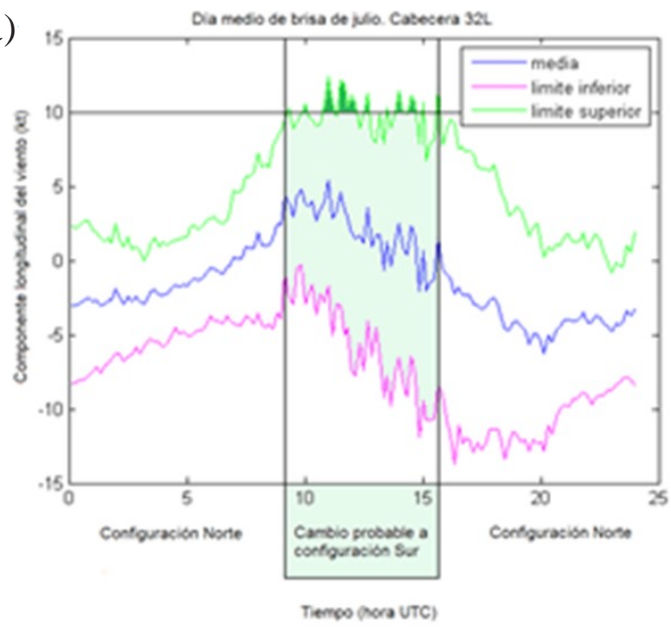

b)

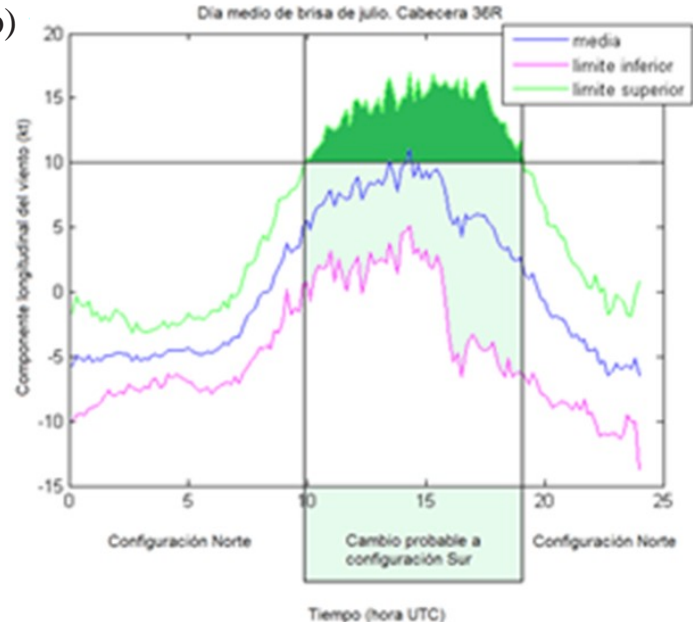

Figura 4. En azul, comportamiento del viento longitudinal medio en a) la cabecera 32L y b) en la cabecera 36R.

Los valores positivos hacen alusión al viento en cola, mientras que los negativos al viento en cara.

Se parte de la hipótesis inicial de configuración norte. Los límites superiores e inferiores son las desviaciones típicas del viento medio. Lo más importante es la región en la que se supera el umbral de 10 kt de viento en cola. Los límites superior e inferior representan la desviación típica. Fuente: AEMET.

\section{CIZALLADURA}

La cizalladura vertical en niveles bajos (por debajo de $2000 \mathrm{ft}$ ) es un fenómeno relativamente frecuente en LEMD. Puede aparecer en cualquier situación de viento fuerte pero hay un caso de especial relevancia y frecuencia cuando, en situación de estabilidad, alguna de las componentes del viento es perpendicular al sistema Central (sierra de Guadarrama). En este caso se produce un fenómeno meteorológico de mesoescala denominado dipolo orográfico.

a) Dipolo con viento del $\mathrm{N}$

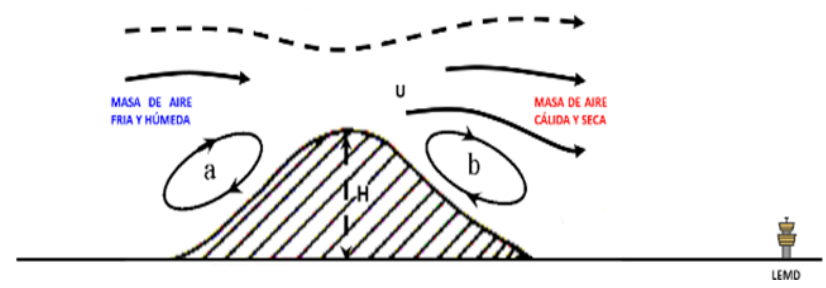

b)

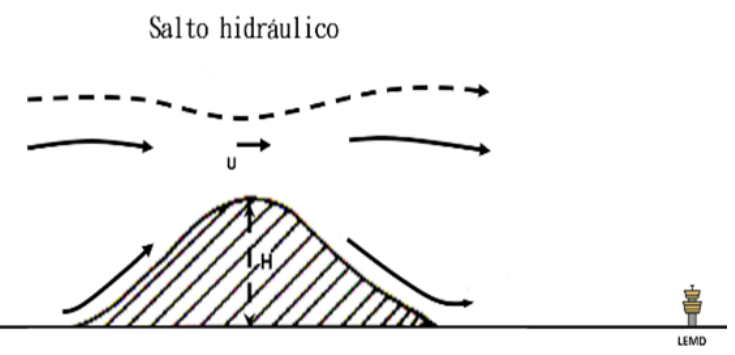

Figura 5. Modelo conceptual del dipolo de componente N en LEMD (corte transversal Norte-Sur).

a) Localización de la mesoalta a barlovento y mesobaja a sotavento.

b) La rotura con el correspondiente salto hidráulico. Fuente: adaptación de Hunt y Simpson. 
El dipolo orográfico consiste en una redistribución del campo de presión y viento, una mesoalta de presión que se forma a barlovento y una mesobaja a sotavento. Este hecho trae consigo una redistribución del campo de viento que hará que los vientos se frenen a barlovento y converjan en el abrigo de sotavento. En niveles por encima del obstáculo montañoso se producirá el fenómeno de ondas de montaña que, como sabemos, pueden propagarse en la vertical desde superficie hasta la tropopausa, lo que provoca turbulencias de aire claro.

Si la retención cesa puede producirse el salto hidráulico que hará que el viento fluya con fuerza por la ladera de sotavento. No obstante, la retención puede volver a ocurrir una y otra vez por lo que la predicción del fenómeno se hace complicada.

Como se comentó anteriormente, LEMD se localiza en la vertiente sur del sistema Central. De entre los dos escenarios de dipolo orográfico plausibles (flujo de componente N con LEMD a sotavento y flujo de componente S con LEMD a barlovento), el de sur es el que tiene mayor impacto en la operatividad del aeródromo (fig. 6).

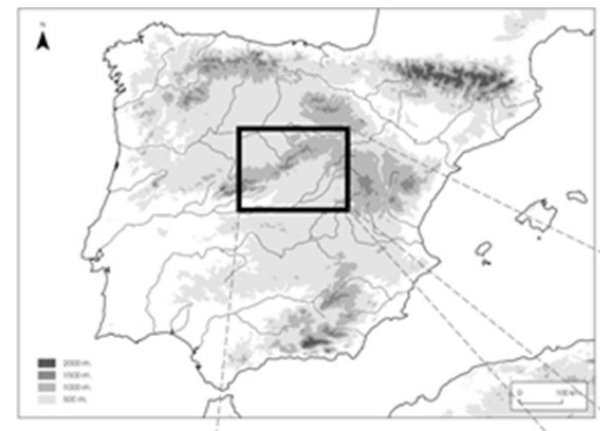

Figura 6.

Sobre una topografía de Madrid se representa, a) el dipolo de sur y

b) el dipolo de norte.

Las flechas azules indican el flujo de aire en superficie.

a)

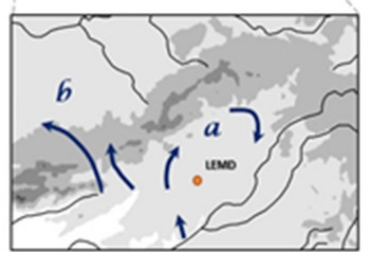

b)

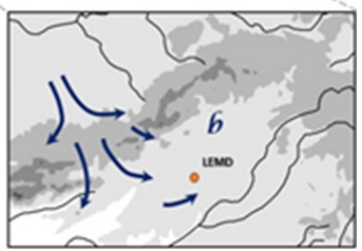

En condiciones de estabilidad y estableciéndose un flujo sinóptico de S, en LEMD el viento es flojo e incluso de componente $\mathrm{N}$ ya que sobre este se halla la mesoalta de barlovento. En este estado de cosas, LEMD se hallará entonces en configuración norte pero, a pocos cientos de metros por encima, soplará viento del S que puede superar holgadamente los $10 \mathrm{kt}$ de cola.

En la figura 7 se muestra un episodio que tuvo lugar el 19 de abril de 2016. Puede apreciarse que el viento era del $\mathrm{S}$ en todos los niveles excepto en los más próximos a la superficie, que eran del $\mathrm{N}$.

La situación meteorológica que da origen al dipolo de sur en Barajas se da siempre que se aproxima un frente frío desde el W.

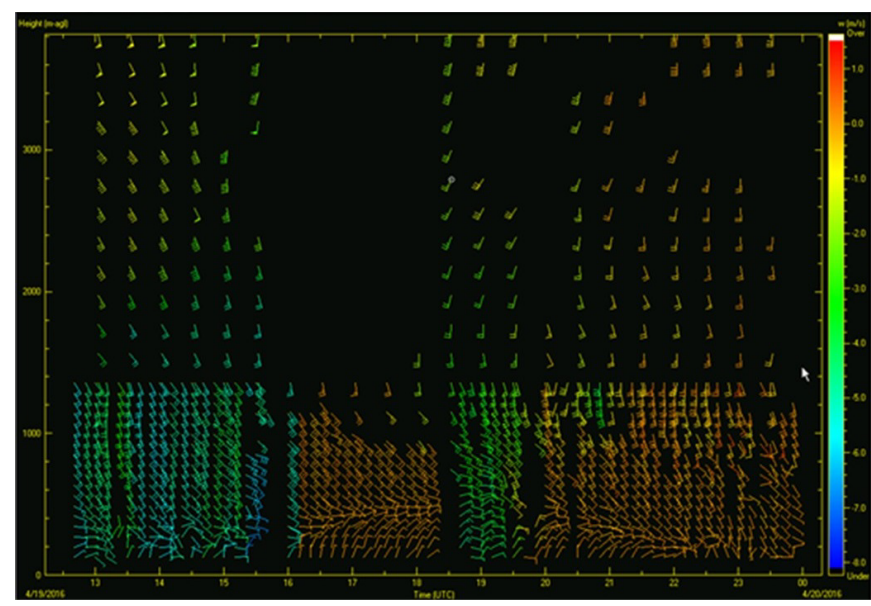

Figura 7.

Perfil vertical del viento en Barajas el 19-4-2016.

En el eje de abscisas se representan las

horas (UTC) y en el de abscisas la altura vertical desde el suelo. La intensidad del viento se denota en barbas mientras que los colores representan la componente vertical. Por debajo de $1200 \mathrm{~m}$ los datos son diezminutales mientras que por encima semihorarios (Pelacho Aja, Cano Espadas y Ayensa Ramirez, 2014). 


\section{NIEBLAS}

La niebla es uno de los fenómenos meteorológicos que mayor incidencia tiene sobre las operaciones en LEMD. Aunque puede aparecer en varias situaciones meteorológicas, en este punto se describirá las dos más habituales:

- Nieblas de río.

- Nieblas por advección cálida desde el Mediterráneo y/o el Atlántico (CANo y TerRAdELlAs, 2006).

Dado que las nieblas en LEMD suelen formarse de madrugada (mínimo de operaciones) y disiparse a medio día (máximo de operaciones), pronosticar la hora de disipación es crítica ya que puede ocasionar acumulaciones en el espacio aéreo que podrían evitarse manteniendo los aviones en los aeropuertos de origen.

\subsection{Nieblas producidas por advección cálida desde el Mediterráneo}

En condiciones de estabilidad y cielos despejados, cuando un flujo sinóptico de procedencia del Mediterráneo llega hasta el valle del Tajo, atravesando previamente el portillo orográfico de Almansa (Albacete) y La Mancha, se produce un extenso banco de niebla con una forma muy característica de luna en $C$ (figura 8) el cual se puede localizar en las inmediaciones de LEMD (CANO, PALACIO, TÉllez et al., 2001). Es muy común que solo afecte a las cabeceras 32 que, en configuración preferente de LEMD, son las de arribada. No obstante, aunque ninguna cabecera se halle afectada por la niebla, las aeronaves encontrarán el banco en la senda de aproximación durante mucha parte del recorrido.
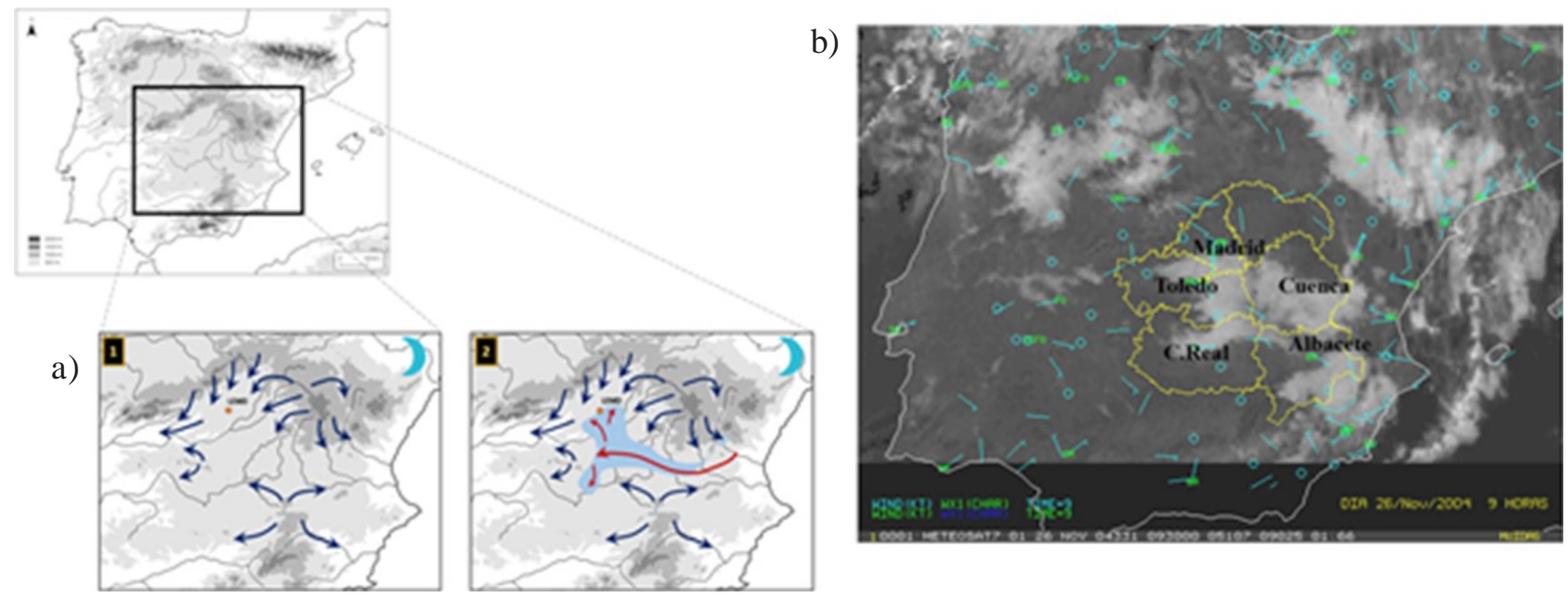

Figura 8. a) Modelo conceptual de formación de nieblas en la meseta meridional. Sobre un mapa con la orografía se representa el flujo catabático (flechas negras) y el flujo del Mediterráneo sobre el banco de niebla (amarillo). b) Imagen de satélide de las 9 UTC del 26 de noviembre de 2004 sobre la que se han ploteado los vientos y los fenómenos significativos. También se localizan las capitales de las provincias afectadas.

\subsection{Niebla producida por la presencia del río Jarama}

Dado que el río Jarama fluye paralelo a las pistas de LEMD (figura 1), es bastante común que la niebla que induce afecte solamente a las pistas más próximas a este, resultando las otras dos sin indidencias. Como el TAF y el TREND van dirigidos a la «visibilidad prevalente» (mejor visibilidad de la mitad del aeródromo), es difícil programar las operaciones en esta situación. Sería deseable establecer procedimientos por fases para poder operar en la mitad del aeródromo que goza de buena visibilidad. Para ello, habría que elaborar un pronóstico para cada una de las pistas. 


\section{TORMENTAS}

Por lo general, las tormentas constituyen el principal fenómeno de impacto sobre un aeródromo. Sus efectos adversos pueden sentirse tanto en este como en sus proximidades. En este punto, se describirá una situación muy habitual en LEMD.

En LEMD es frecuente que las tormentas aparezcan cuando se opera en configuración sur. Estas se forman en el sistema Central (sierra de Guadarrama) a medio día, desplazándose a lo largo de la tarde, valle abajo, hacia Madrid (CANO, PALACIO y SÁnchez, 1996). Aunque no lleguen al mismo aeródromo, sus efectos pueden sentirse a distancia, en forma de fuertes rachas provocadas por los reventones (figura 9).
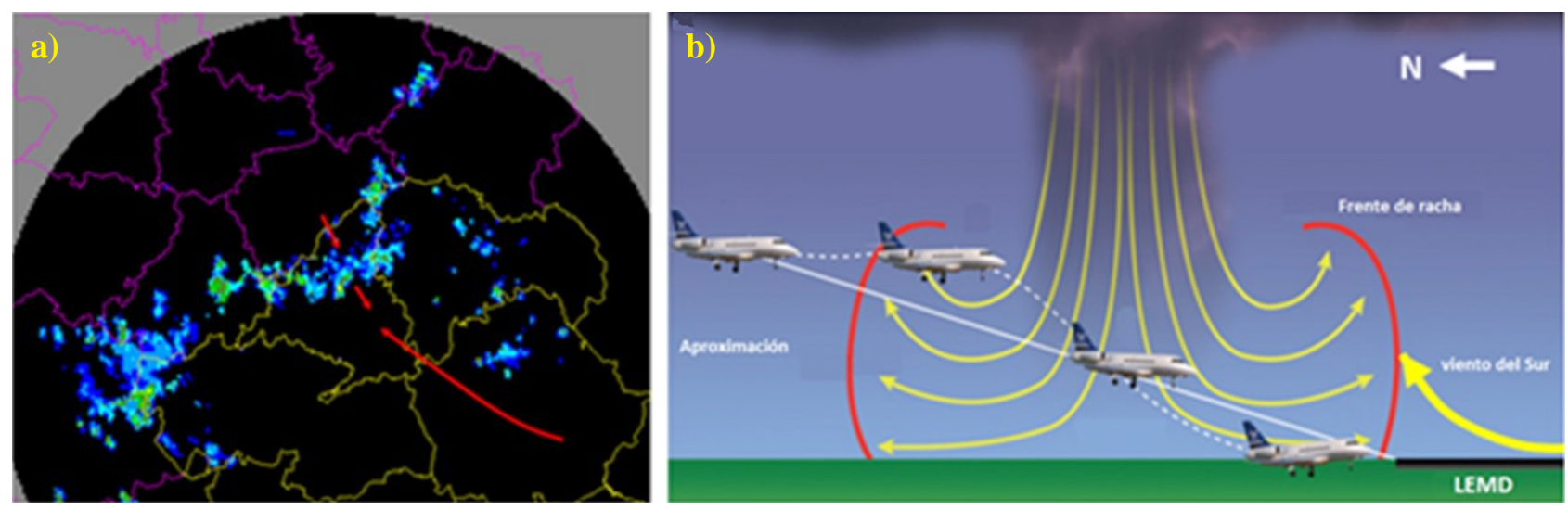

Figura 9. a) Imagen PPI del radar de Madrid en el momento que una línea de tormentas avanza desde la Sierra al aeropuerto (con flechas rojas se representa la dirección del flujo de viento).

b) Se representa el esquema de un reventón convectivo localizado al norte del aeropuerto que se halla en configuración sur. Fuente: AEMET.

En este caso, LEMD puede encontrarse en la zona de convergencia del frente de racha producido por las tormentas por lo que tendrá un viento de componente $\mathrm{N}$ en las cabeceras 18 y de componente $\mathrm{S}$ en las cabeceras 32, lo que constituye una situación difícil para establecer una adecuada configuración de operación. Si las tormentas se encuentran fuera de un radio de $8 \mathrm{~km}$, no aparecerán reflejadas en el METAR ni tampoco en el TAF (ambos, productos de aeródromo dirigidos a la navegación aérea). Este hecho no quiere decir que no se observen sus efectos.

\section{NIEVE}

La nieve es un fenómeno poco frecuente en LEMD. Según el Banco de Datos Climatológico de AEMET (periodo de referencia 1981-2010), suele ocurrir, por término medio unos 2,6 días a lo largo del año. Muy extrañas son aquellas situaciones en las que cubren el suelo, provocando una contaminación de las pistas.

LEMD dispone de una predicción a tres días, además de los mensajes ordinarios TAF, WORNING completo que incluye probabilidad de ocurrencia y espesor del depósito, TREND y METAR.

Para llevar a cabo las tareas de limpieza de las pistas y uso de fundentes es de vital importancia la predicción de muy corto plazo (TREND) tanto para el comienzo como la finalización de cada episodio.

Desde un punto de vista meteorológico, las nevadas más importantes en LEMD ocurren cuando los vientos templados de componente W-SW (e incluso SE) se desplazan sobre el aire seco y frío, con heladas que, o 
bien dejó estancado antes, durante varios días, el anticiclón, o bien penetra desde el norte por los niveles cercanos a superficie.

Por otra parte, el aguanieve (RASN) no es motivo de aviso pero puede ocasionar los mismos problemas o incluso aún mayores si se deposita sobre la pista ya que su retirada es más complicada que en el caso de la nieve.

Dado que las actuaciones del aeródromo en situaciones de nevadas incluyen las carreteras de acceso al aeropuerto, es muy importante la coordinación con el Ayuntamiento. Así, los avisos de aeródromo deben estar coordinados con los avisos de Protección Civil.

\section{CONCLUSIONES}

Las situaciones meteorológicas que tienen impacto en la operatividad de LEMD no tienen por qué vincularse a lo que socialmente se denomina «tiempo adverso». Hay muchas situaciones de «buen tiempo» que son precursoras de decisiones técnicas por parte del gestor aeroportuario o del centro de control de área. Por ejemplo, el cambio de configuración de las pistas se produce como consecuencia de la brisa de montaña.

El impacto no solo depende del escenario meteorológico en el que se halle inmerso LEMD sino también de la fase del día y del número de operaciones, tráfico de pasajeros o carga aérea. En este sentido, la baja visibilidad por la formación de nieblas, que suele ocurrir durante la madrugada cuando el volumen de tráfico es bajo, puede ser crítica si su disipación se alarga hasta las primeras horas del día.

\section{REFERENCIAS}

Cano, D., Palacio, J. I. y Sánchez, T, 1996. Estudio de dos situaciones convectivas en el CMT en Madrid y CLM. IV Simposio Nacional de Predicción. INM.

Cano, D., Palacio, J. I., Téllez, B. y Arias, P., 2001. Estudio de las zonas más favorables para el desarrollo de tormentas en la Meseta Meridional. El Tiempo del Clima, 313-324, AEC.

Téllez, B., Roa, A., Palacio, I. y CAno, D., 2001. Uso de modelos conceptuales de mesoescala regionalizados para el estudio de una situación convectiva. V Simposio Nacional de Predicción. INM.

CAno, D., Palacio, J. I., Téllez, B. et al., 2001. Importancia de los flujos catabáticos en las predicciones para el aeropuerto de Madrid-Barajas. V Simposio Nacional de Predicción. INM.

Cano, D., Palacio, J. I., Téllez, B. et al., 2001. Estudios de nieblas realizados en el CMT en Madrid y Castilla-La Mancha. V Simposio Nacional de Predicción. INM.

Palacio, J. Cano, D. y Téllez, B., 2004. Convección estival en las comunidades de Madrid y Castilla-La Mancha. XXVIII Jornadas científicas. AME, Badajoz.

CANo, D. y Terradellas, E., 2006. Behavior of the humidity and the horizontal mass convergence in the surroundings of the Madrid Barajas airport in days with mountain breezes. COST 722.

TeRradellas, E. y CANO, D., 2007. Implementation of a single-column model for fog and low clouds forecasting at central spanisch airports. Pure appl. geophys., 164: 1327. https://doi.org/10.1007/s00024-007-0221-8.

CANO, D. et al., 2006. Caracterización de la capa límite en Madrid en situaciones de estabilidad atmosférica. $5^{\mathrm{a}}$ Asamblea hispano-portuguesa de geodesia y geofísica.

Pelacho Aja, M. A., Cano Espadas, D. y Ayensa Ramírez, E., 2014. Uso operacional de un perfilador de viento para la meteorología aeronáutica. XXXIII Jornadas Científicas. AME. 